\title{
A New Proof of a Contrast Function for Bounded Component Analysis and Further Analysis
}

\author{
Wei Gao ${ }^{\mathrm{a}, *}$, Shen Fan ${ }^{\mathrm{b}}$, Roberto Togneri ${ }^{\mathrm{a}}$, Victor Sreeram ${ }^{\mathrm{a}}$ \\ ${ }^{a}$ School of Electrical Electronic and Computer Engineering, The University of Western \\ Australia, Perth 6009, Australia. \\ ${ }^{b}$ College of Science, China University of petroleum (Beijing), Beijing 102200, China
}

\begin{abstract}
Bounded Component Analysis (BCA) solves the Blind Source Separation (BSS) problem based on geometric assumptions. This paper introduces a new proof of a BCA contrast function, derived from elementary matrices, Gauss-Jordan elimination and convex geometry. The new proof and further analysis provide additional insight into a key assumption of BCA. In addition, an interpretation is presented to clarify one of the limitations of the instantaneous BCA algorithm. Experiments on audio sources support our analysis.
\end{abstract}

Keywords: bounded component analysis, blind source separation, independent component analysis, elementary matrix, convex geometry, audio signals.

\section{Introduction}

As the name "blind" suggests, Blind source separation (BSS) aims to recover the sources from mixtures of the sources only, without prior information of the sources and the way the sources were mixed [1]. The "blind" feature not only leads to a broad variety of applications in practice, such as

\footnotetext{
*Corresponding author

Email addresses: wei.gao@research.uwa.edu.au (Wei Gao), fans@cup.edu.cn (Shen Fan), roberto.togneri@uwa.edu.au (Roberto Togneri), victor.sreeram@uwa.edu.au (Victor Sreeram)

${ }^{1}$ The work of Shen Fan was supported by Science Foundation of China University of Petroleum, Beijing (No.YJRC-2011-14, No.FZG-2011-01).
} 
speech processing, digital communications, image processing and chemometrics, etc., but also makes BSS an exciting challenge as an ill-posed problem [2]. Prior information is generally replaced by some assumptions. The more stringent the assumptions, the narrower the applicability [3], but the easier the BSS problem. There is no BSS algorithm that can be universally used to solve all problems.

A wide variety of assumptions and corresponding algorithms have been developed in different application fields. Independent Component Analysis (ICA) algorithms can be considered as the representation of BSS solutions in the 1990's, proposed in the field of neural networks [4] [5]. Rooted in statistically independent or uncorrelated assumptions of the sources, numerous ICA algorithms of higher order statistics (HOS) and second order statistics (SOS) were developed [6]. For example, SOBI [7], STOTD [8] and JADE [9][10] combine Jacobi rotation with second-order, third-order and fourthorder cumulants, respectively. For computational and conceptual simplicity, BSS solutions were obtained by optimizing a contrast function, for example FastICA [11], a classical and implementable ICA algorithm.

ICA algorithms were further developed by incorporating geometric techniques into the implementation of the BSS algorithm. For nonstatioanary sources, a new joint diagonalization procedure was established based on the principles of maximum likelihood and minimum mutual information [12]. To deal with data contamination, the gamma-ICA method with better robustness was proposed, and implemented by a geometrical framework based on gradient flows on a special orthogonal matrix [13]. The minimum-range approach investigates bounded sources, and geometric interpretation is exploited for contrast maximization over the group of special orthogonal matrices [14]. Similarly, under a certain boundedness assumption of sources, minimization of the infinity norm approach is centered around the basic geometric fact of independent vectors [15]. The restrictions of statistical independence of the sources can be relaxed to partial correlation, a constrast function was proposed by combining geometric techuniques [16].

However, some realistic sources are dependent or correlated. To replace the restrictions of statistical independence, various assumptions and algorithms were proposed, such as Non-negative Matrix Factorization (NMF) and Sparse Component Analysis (SCA) [2].

Compared with NMF and SCA, Bounded Component Analysis (BCA) is a relatively new avenue for BSS [2] without the assumption of independence of the sources. The BCA proposed by Cruces [17], relies on the assump- 
tions of compactness and Cartesian decomposition of the convex support of the vector of the sources. Still based on the convex hull of the sources, [18] developed BCA algorithms by considering the underdetermined mixtures. An alternative framework proposed by Erdogan utilises the principal hyperellipsoid and the bounding hyper-rectangle of the sources [19], which supports Cruces's idea that BCA can be considered as a more general approach, covering ICA as a special case for bounded sources [17]. Then, Erdogan's BCA algorithms were further developed for separating convolutive mixtures [20][21]. Furthermore, sparse BCA algorithm was proposed to consider the sparsity of the sources [22]. Recently, a stationary point for instantaneous BCA algorithms was analysed [23]. Although BCA algorithms have attracted much attention and developed quickly, there is still a question whether BCA should be considered as a more general approach than ICA. In this paper, we will show that BCA should be considered as a complementary approach to ICA, rather than a more general approach covering ICA.

Cruces [17] assumed the following three properties to ensure the separability:

P1) Compactness and nondegeneracy of the sources: all the sources are nondegenerate ${ }^{2}$ random variables of compact support.

P2) Cartesian decomposition of the convex support of the sources: $\mathbb{S}_{\check{S}}=$ $\mathbb{S}_{\check{S}_{1,:}} \otimes \cdots \otimes \mathbb{S}_{\check{S}_{p:}}$, where $\otimes$ denotes Cartesian product, $\mathbb{S}_{\check{S}}$ denote the convex hull of the support of the sources, and $\mathbb{S}_{\check{S}_{i:}}$ denote the convex hull of the support of the $i$ th source.

P3) Lossless mixing: the mixing matrix is full-column rank.

On the basis of P1)-P3), in [19] one more assumption A1) was introduced: $S$ contains the vertices of its bounding hyper-rectangle.

Although [19] have provided a proof of the contrast function (4), it lends little insight into the condition when assumption A1) is not satisfied. This paper not only presents a new proof of the contrast function (4) in Section 2 , but also in Section 3 provides a more in-depth analysis and insight into the following five aspects regarding BCA:

(i) Theoretical discussion on the link between A1) and P2);

\footnotetext{
${ }^{2} \mathrm{~A}$ random variable can be considered degenerate if the support of its p.d.f consist in a single point.
} 
(ii) Although BCA is named by the boundedness of the sources $\mathbf{P 1}$ ), the key and stringent assumption of BCA is Cartesian decomposition of the sources, i.e. P2);

(iii) A1) is likely to be stringent in some practical applications;

(iv) Interpretation on why the BCA algorithm suffers when A1) is not satisfied;

(v) Experiments show how the instantaneous BCA algorithm suffers when applied to blind audio source separation.

The analysis and experiments provide a comprehensive recognition of BCA, not only advantages but also limitations. Equipped with the comprehensive recognition of BCA, readers have more opportunities to select a suitable BSS algorithm.

The remainder of this paper is organised as follows: Section 2 presents the BCA models and notation, as well as some preliminaries; Section 3 provides our proof of Erdogan's BCA contrast function; the BCA assumptions are further analysed in Section 4, and supported by numerical experiments; finally, our conclusion is presented in Section 5 .

\section{Background}

\subsection{Models and Notation}

Consider an instantaneous real-valued BSS model without noise. Observed signals $Y \in \mathbb{R}^{q \times L}$ are linear instantaneous mixtures of the sources $S \in \mathbb{R}^{p \times L}$ such that $Y=H S$, where $H \in \mathbb{R}^{q \times p}$ denotes the mixing matrix. Here $L, q$ and $p$ indicate the number of samples, mixtures and sources, respectively. Note that from the theoretical point of view $L$ can be assumed to be infinite, but in practical applications $L$ is a finite integer. BSS is concerned with finding a separating matrix $W \in \mathbb{R}^{p \times q}$ to obtain the recovered sources $Z=W Y=G S$, where $G=W H$ represents the overall mapping. The $L$ samples of the $i$ th source are expressed as $S_{i,:}$, that is the $i$ th row of $S$. Similarly, $Z_{i,:}$ and $(\cdot)_{i, \text { : }}$ denote the $i$ th row of $Z$ and the enclosed matrix, respectively. Each sample of the sources are expressed as $S_{:, j}$, that is the $j$ th column of $S$. Similarly, $(\cdot)_{:, j}$ denote the $j$ th column of the enclosed matrix.

Let

$$
\hat{\mathbf{l}}(S)=\left[\begin{array}{c}
\min _{k \in\{1, \cdots, L\}} S_{1, k} \\
\vdots \\
\min _{k \in\{1, \cdots, L\}} S_{p, k}
\end{array}\right]
$$




$$
\hat{\mathbf{u}}(S)=\left[\begin{array}{c}
\max _{k \in\{1, \cdots, L\}} S_{1, k} \\
\vdots \\
\max _{k \in\{1, \cdots, L\}} S_{p, k}
\end{array}\right]
$$

denote the vectors containing minimum and maximum values for the rows of $S$, respectively. The bounding hyper-rectangle of $S$ can be defined as

$$
\widehat{B}(S)=\{\mathbf{q}: \hat{\mathbf{l}}(S) \leqslant \mathbf{q} \leqslant \hat{\mathbf{u}}(S)\}
$$

Thus, $\widehat{B}(S)$ is defined as the minimum volume box covering all samples of $S$ and aligning with the coordinate axes. Vertices of $\widehat{B}(S)$ are determined by the minimum and maximum of each row of $S$. Similarly, $\widehat{B}(Z)$ denotes the bounding hyper-rectangle of $Z$.

Additionally, $\operatorname{cov}(\cdot), \operatorname{abs}(\cdot)$ and $\operatorname{det}(\cdot)$ express the covariance matrix, the absolute value and the determinant, respectively. The center of the principal hyper-ellipsoid of $S$ is given by the sample mean of $S$, which is defined as

$$
\hat{\mu}(S)=\frac{1}{L} \sum_{k=1}^{L} S_{:, k} .
$$

The principal semiaxes directions are determined by the eigenvectors of the sample covariance matrix of $S$ as

$$
\operatorname{cov}(S)=\frac{1}{L} \sum_{k=1}^{L}\left(S_{:, k}-\hat{\mu}(S)\right)\left(S_{:, k}-\hat{\mu}(S)\right)^{T} .
$$

Then, principal hyper-ellipsoid of $S$ can be defined as

$$
\widehat{\varepsilon}(S)=\left\{\mathbf{q}:(\mathbf{q}-\hat{\mu}(S))^{T} \operatorname{cov}(S)^{-1}(\mathbf{q}-\hat{\mu}(S)) \leqslant 1\right\} .
$$

Under the above four assumptions, Erdogan [19] proposed a family of contrast functions based on:

$$
\operatorname{maximize}\left\{\frac{\operatorname{vol}[\widehat{\varepsilon}(Z)]}{\operatorname{vol}[\widehat{B}(Z)]}=\frac{\operatorname{vol}[\widehat{\varepsilon}(G S)]}{\operatorname{vol}[\widehat{B}(G S)]}\right\},
$$

where vol[.] denotes the volume. The volume of the principal hyper-ellipsoid of $Z$ is:

$$
\operatorname{vol}[\widehat{\varepsilon}(Z)]=C_{p} \sqrt{\operatorname{det}(\operatorname{cov}(Z))}=\operatorname{vol}[\widehat{\varepsilon}(G S)]=\operatorname{abs}(\operatorname{det}(G)) \operatorname{vol}[\widehat{\varepsilon}(S)],
$$


where $C_{p}=\pi^{\frac{p}{2}} / \Gamma\left(\frac{p}{2}\right)$, and $\Gamma(\cdot)$ denotes the Gamma function. The volume of the bounding hyper-rectangle of $Z$ can be expressed compactly as:

$$
\operatorname{vol}[\widehat{B}(Z)]=\prod_{i=1}^{p}\left\{\max \left[Z_{i,:}\right]-\min \left[Z_{i,:}\right]\right\}=\operatorname{vol}[\widehat{B}(G S)]
$$

\subsection{Preliminaries}

In linear algebra, there are three types of elementary matrices $E_{1}, E_{2}$ and $E_{3}$, which are defined from the identity matrix $I$ as follows:

$$
E_{1}\left(I_{u} \leftrightharpoons I_{v}\right)=\left[\begin{array}{lllllll}
1 & & & & & & \\
& \cdot & & & & & \\
& 0 & & 1 & \\
& & & & & & \\
& 1 & & 0 & & \\
& & & & \cdot & \\
& & & & & 1
\end{array}\right]
$$

where $I_{u}$ (the $u$ th row of $I$ ) is switched with $I_{v}$ (the $v$ th row of $I$ );

$$
E_{2}\left(h I_{u}\right)=\left[\begin{array}{cccccc}
\cdot & & & & & \\
& 1 & & & \\
& h & & \\
& & 1 & \\
& & & \cdot
\end{array}\right]
$$

where $I_{u}$ is multiplied by a non-zero scalar $h \neq 0$;

$$
E_{3}\left(k I_{v} \rightarrow I_{u}\right)=\left[\begin{array}{cccccc}
\cdot & & & & & \\
& 1 & & & k & \\
& & \cdot & & & \\
& & & 1 & \\
& & & & \cdot
\end{array}\right]
$$

where $k \neq 0$ and $I_{v}$ multiplied by $k$ is added to $I_{u}$. For brevity of notation, hereinafter elementary matrices are of appropriate dimension for matrix multiplications, and the parameters for the elementary matrices are omitted. Left multiplication by $E_{1}, E_{2}$ and $E_{3}$ correspond to reflection mapping, scale mapping and shear mapping, respectively. 
A convex hull of a set $X=\left\{X_{:, 1}, \cdots, X_{:, L}\right\}$, where $X_{:, i} \in \mathbb{R}^{N}$ denotes a point, can be defined as

$$
\operatorname{Conv}(X)=\left\{\sum_{i=1}^{L} X_{:, i} \theta_{i} \mid \sum_{i=1}^{L} \theta_{i}=1 \wedge\left(\forall i: \theta_{i} \geqslant 0\right)\right\}
$$

Roughly speaking, $\operatorname{Conv}(X)$ is the smallest convex set that contains $X$. For a compact finite set $X$, if $A$ is a linear mapping (equivalent to a linear matrix in linear algebra), then $\operatorname{Conv}(A(X))=A(\operatorname{Conv}(X))$ and $\operatorname{vol}[\operatorname{Conv}(A(X))]=$ $\operatorname{abs}(\operatorname{det}(A)) \operatorname{vol}[\operatorname{Conv}(X)][24][25]$.

\section{New Proof of Erdogan's BCA}

The new proof presented here assumes real-valued signals. The extension to complex-valued signals is relatively straightforward, albeit cumbersome, requiring the $\max [\cdot]$ and $\min [\cdot]$ operations to be carried out on the real-part and imaginary-part of signals separately. This essentially treats complexvalued signals as paired real-valued signals. In this section, we first analyse the mixing matrix and the overall mapping. Then our proof is presented in three steps. Finally, our remarks following our proof are presented.

\subsection{Analysis of the Overall Mapping}

Note that the geometric concepts $\widehat{B}(S)$ and $\operatorname{Conv}(S)$ are formulated by viewing the matrix $S$ as a set composed of the columns of $S$, i.e. $S=$ $\left\{S_{:, 1}, \cdots, S_{:, j}, \cdots, S_{: L L}\right\}$, where $S_{:, j}$ represents a column of the matrix $S$. This view has been the basis of a scatter plot in the BSS problem [2]. We exploit this view and geometric concepts to analyse the BCA contrast function.

Under P1), "compactness" implies that each source $S_{i, \text { : }}$ attains a finite maximum and minimum; "nondegeneracy" implies that for each source $S_{i, \text { : }}$ its maximum is greater than its minimum. It follows that $\max \left[S_{i,:}\right]-$ $\min \left[S_{i,:}\right]>0, i \in\{1, \cdots, p\}$. Hence, substituting $E_{1}$ in (7) into (6),i.e., $G=E_{1}$, we get for any $E_{1}$

$$
\begin{aligned}
\operatorname{vol}\left[\widehat{B}\left(E_{1} S\right)\right] & =\prod_{i=1}^{p}\left\{\max \left[\left(E_{1} S\right)_{i,:}\right]-\min \left[\left(E_{1} S\right)_{i,:}\right]\right\} \\
& =\prod\left\{\max \left[S_{i,:}\right]-\min \left[S_{i,:}\right]\right\}, i=1, \cdots, v, \cdots, u, \cdots, p \\
& =\prod\left\{\max \left[S_{i,:}\right]-\min \left[S_{i,:}\right]\right\}, i=1, \cdots, u, \cdots, v, \cdots, p \\
& =\operatorname{vol}[\widehat{B}(S)] .
\end{aligned}
$$


Given $\operatorname{abs}\left(\operatorname{det}\left(E_{1}\right)\right)=1$, we can state more generally that

$$
\operatorname{vol}\left[\widehat{B}\left(E_{1} S\right)\right]=\operatorname{abs}\left(\operatorname{det}\left(E_{1}\right)\right) \operatorname{vol}[\widehat{B}(S)], \forall E_{1} .
$$

In the same way, substituting $E_{2}$ in (8) into (6), and noting abs $\left(\operatorname{det}\left(E_{2}\right)\right)=$ $\operatorname{abs}(h)$, we get

$$
\operatorname{vol}\left[\widehat{B}\left(E_{2} S\right)\right]=\operatorname{abs}(h) \operatorname{vol}[\widehat{B}(S)]=\operatorname{abs}\left(\operatorname{det}\left(E_{2}\right)\right) \operatorname{vol}[\widehat{B}(S)], \forall E_{2} .
$$

Note that the elementary matrix has the property of full rank. Thus, if we consider an elementary matrix as the simplest mixing matrix $H$ or the simplest overall mapping $G$, the equalities of (10) and (11) rely on P1) and P3).

Under $\mathbf{A} \mathbf{1}$ ) and $\mathbf{P} \mathbf{1}$ ), the bounding hyper-rectangle $\widehat{B}(S)$ is a nondegenerate $p$-dimensional hyper-rectangle with edges aligning with coordinate axes in geometry. Under P3), $G$ is a full-rank square matrix, and it follows that $G$ is invertible. Therefore, under $G$ the bounding hyper-rectangle $\widehat{B}(S)$ is mapped to a nondegenerate $p$-dimensional hyper-parallelogram $G \widehat{B}(S)$, whose edges may not align with coordinate axes. We consider the constraints on $G$ that make "align with coordinate axes" preserved, in the following Lemma.

Lemma 1. Under an invertible linear $G$, if $G \widehat{B}(S)$ is also a bounding hyperrectangle with edges aligning with coordinate axes, then there exist $D$ and $P$ such that $G=D P$, where $D$ is an invertible square diagonal matrix, and $P$ is an invertible square permutation matrix.

Proof. The $p$ column vectors $e_{1}=[1,0, \cdots, 0]^{T}, \cdots, e_{p}=[0, \cdots, 0,1]^{T}$ denote $p$ unit-coordinate vectors of $\mathbb{R}^{p}$, such that $e_{k}$ is the $k$ th column of an identity matrix $I \in \mathbb{R}^{p \times p}$. Since the edges of the bounding hyper-rectangle are aligned with the coordinate axes, for any vertex $V_{0}$ of $\widehat{B}(S)$, there exists an edge $\overrightarrow{V_{0} V_{k}}$ starting from $V_{0}$ to another vertex $V_{k}$ that is parallel to $e_{k}$, $k=1, \cdots, p$. Let $a_{k}$ denote the length of $\overrightarrow{V_{0} V_{k}}$, and we then have

$$
\overrightarrow{V_{0} V_{k}}=a_{k} e_{k}, k=1, \cdots, p,
$$

where $a_{k} \neq 0$ by $\left.\mathbf{P} 1\right)$.

Denote the images of $V_{0}, V_{1}, \cdots, V_{p}$ under $G$ as $V_{0}^{\prime}, V_{1}^{\prime}, \cdots, V_{p}^{\prime}$, which are still the vertices of $G \widehat{B}(S)$, given $G$ is an invertible linear mapping by P3). Then, the mapped edge

$$
\overrightarrow{V_{0}^{\prime} V_{k}^{\prime}}=G \overrightarrow{V_{0} V_{k}}, k=1, \cdots, p .
$$


If $G \widehat{B}(S)$ is also a $p$-dimensional bounding hyper-rectangle, which means each edge of $G \widehat{B}(S)$ is aligned with a coordinate axis, there exists $e_{i_{k}}$ parallel to $\overrightarrow{V_{0}^{\prime} V_{k}^{\prime}}$, where $i_{k} \in\{1, \cdots, p\}$ is the index of the unit-coordinate vector, i.e., the linear mapping $G$ maps $e_{k}$ to $e_{i_{k}}$. Denote $b_{i_{k}}$ the length of the edge $\overrightarrow{V_{0}^{\prime} V_{k}^{\prime}}$, so

$$
\overrightarrow{V_{0}^{\prime} V_{k}^{\prime}}=b_{i_{k}} e_{i_{k}}, k=1, \cdots, p .
$$

Since $G$ is an invertible matrix, $b_{i_{k}} \neq 0, \forall k \in\{1, \cdots, p\}$, and $i_{1}, i_{2} \cdots i_{p}$ is a permutation of $1,2, \cdots, p$.

Combining (12),(13) and (14) together, we have $G \overrightarrow{V_{0} V_{k}}=a_{k} G e_{k}=$ $b_{i_{k}} e_{i_{k}}, k=1, \cdots, p$, and hence we get $G e_{k}=\frac{b_{i_{k}}}{a_{k}} e_{i_{k}}$. Given $e_{k}$ is the $k$ th column of $I$, that is $\left[e_{1}, e_{2}, \cdots, e_{p}\right]=I$, we have

$$
G=G\left[e_{1}, e_{2}, \cdots, e_{p}\right]=\left[\begin{array}{cccc}
\frac{b_{i_{1}}}{a_{1}} & & & 0 \\
& \cdot & & \\
& \frac{b_{i_{k}}}{a_{k}} & & \\
0 & & & \frac{b_{i_{p}}}{a_{p}}
\end{array}\right]\left[e_{i_{1}}, \cdots, e_{i_{p}}\right],
$$

Let $D$ denote the square diagonal matrix

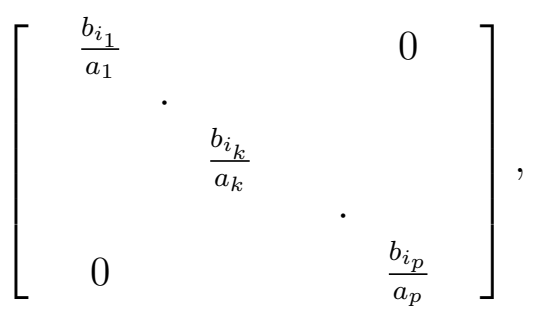

and $P$ denote the square permutation matrix $\left[e_{i_{1}}, \cdots, e_{i_{p}}\right]$. Since $G$ is an invertible matrix, $b_{i_{k}} \neq 0, \forall k \in\{1, \cdots, p\}$, and $i_{1}, i_{2} \cdots i_{p}$ is a permutation of $1,2, \cdots, p$. Thus, $G=D P$ where $D$ is an invertible square diagonal matrix, and $P$ is an invertible square permutation matrix.

\subsection{New Proof of the BCA Contrast Function}

Our proof of Erdogan's contrast function (4) can be divided into three steps: Step1 proves that if $G$ is a perfect solution, then $\operatorname{vol}[\widehat{B}(G S)]=$ $\operatorname{abs}(\operatorname{det}(G)) \operatorname{vol}[\widehat{B}(S)]$; Step2 proves that if the condition in Step1 holds, then $G$ is a perfect solution; Step3 combines Step1 and Step2 together as proof of the contrast function (4). 


\section{Proof. Step1}

Under P3), the overall mapping $G$ is a full-rank square matrix, which can be classified into two classes: the perfect solution denoted by $\bar{G}$, and the imperfect solution denoted by $\underline{G}$. Due to unavoidable ambiguities of BSS, if $G$ is up to row permutation and scaling from an identity matrix, $G$ is classified as a perfect solution. It follows that any perfect solution $\bar{G}=D P$ [2], where $D$ is an invertible square diagonal matrix, and $P$ is an invertible square permutation matrix.

According to Gauss-Jordan elimination, $D$ can be simply factorized as a product of finite $E_{2}$ factors, $D \triangleq \prod_{f_{2}=1}^{F_{2}} E_{2}^{f_{2}}$, where the superscript $f_{2}$ denotes the $f_{2}$ th factor, and $F_{2}$ is a non-negative integer denoting the total number of $E_{2}$ factors. Note that $\triangleq$ denotes simply factorized, which requires that every elementary matrix factor impacts different entries of $I$. In other words, any identity matrix and any elementary matrix should not be further factorized to two or more elementary matrices.

Similarly, $P$ can be simply factorized as a product of finite $E_{1}$ factors, $P \triangleq \prod_{f_{1}=1}^{F_{1}} E_{1}^{f_{1}}$, where the superscript $f_{1}$ denotes the $f_{1}$ th factor, and $F_{1}$ is a non-negative integer denoting the number of $E_{1}$ factors. We can state more generally that, if and only if $\bar{G}$ is a perfect solution,

$$
\bar{G} \triangleq \prod_{f=0}^{F} E_{i}^{f}, i \in\{1,2\},
$$

where the superscript $f$ denotes the $f$ th elementary matrix factor, and $F=$ $F_{1}+F_{2}$ denotes the total number of elementary matrix factors. If $F_{1}=0$ and $F_{2}=0, \bar{G}=I$.

By Gauss-Jordan elimination, the total number of simply factorized factors of $G$ is finite. After finite transformations, the equality of (10) and (11) will still hold, under P1) and P3). For the perfect solution $\bar{G}$, substituting (15) into (6), and simplifying sequentially by (10) and (11) together, we get

$$
\begin{aligned}
& \operatorname{vol}[\widehat{B}(\bar{G} S)]=\prod_{f_{2}=1}^{F_{2}} \operatorname{abs}\left(\operatorname{det}\left(E_{2}^{f_{2}}\right)\right) \prod_{f_{1}=1}^{F_{1}} \operatorname{abs}\left(\operatorname{det}\left(E_{1}^{f_{1}}\right)\right) \operatorname{vol}[\widehat{B}(S)] \\
= & \operatorname{abs}(\operatorname{det}(D)) \operatorname{abs}(\operatorname{det}(P)) \operatorname{vol}[\widehat{B}(S)]=\operatorname{abs}(\operatorname{det}(\bar{G})) \operatorname{vol}[\widehat{B}(S)] .
\end{aligned}
$$

\section{Step2}


Under P1), by the definition in (1) (2) and (3), $\widehat{B}(S)$ is an intersection of a lower half-space $\{\mathbf{q} \leqslant \hat{\mathbf{u}}(S)\}$ and an upper half-space $\{\hat{\mathbf{l}}(S) \leqslant \mathbf{q}\}$. Thus, $\widehat{B}(S)$ is a compact convex set that contains $S$. Since the convex hull $\operatorname{Conv}(S)$ is the intersection of all convex sets containing $S, \operatorname{Conv}(S) \subseteq \widehat{B}(S)$. With the addition of A1): $S$ contains the vertices of its bounding hyper-rectangle, and these vertices are the maximum and minimum values of each row of $S$. It follows that the vertices of $\widehat{B}(S)$ are also the vertices of $\operatorname{Conv}(S)$. Hence, the compact convex set $\widehat{B}(S)$ is the smallest convex set that contains $S$, and $\widehat{B}(S)$ is also the convex hull of $S$. This means that under P1) and A1),

$$
\widehat{B}(S)=\operatorname{Conv}(S) \text {. }
$$

By definition, $\widehat{B}(G S)$ is a compact convex set that contains $G S$, so

$$
\operatorname{Conv}(G S) \subseteq \widehat{B}(G S)
$$

Since $G$ is a linear transformation, we have

$$
G \operatorname{Conv}(S)=\operatorname{Conv}(G S) .
$$

Combining (17) (18) and (19) together, we get

$$
G \widehat{B}(S)=G \operatorname{Conv}(S)=\operatorname{Conv}(G S) \subseteq \widehat{B}(G S) .
$$

Then, $\operatorname{vol}[G \widehat{B}(S)] \leqslant \operatorname{vol}[\widehat{B}(G S)]$, where the " = " is of interest. We use contradiction to prove that if $\operatorname{vol}[G \widehat{B}(S)]=\operatorname{vol}[\widehat{B}(G S)]$, then $G \widehat{B}(S)=$ $\widehat{B}(G S)$.

Assume to the contrary that when $\operatorname{vol}[G \widehat{B}(S)]=\operatorname{vol}[\widehat{B}(G S)], G \widehat{B}(S) \neq$ $\widehat{B}(G S)$. By $(20)$, the contrary assumption follows that when $\operatorname{vol}[G \widehat{B}(S)]=$ $\operatorname{vol}[\widehat{B}(G S)], G \widehat{B}(S) \subset \widehat{B}(G S)$. Given $G \widehat{B}(S) \subset \widehat{B}(G S)$, there must exist a vertex $V$ of $\widehat{B}(G S)$ such that $V \notin G \widehat{B}(S)$. On one hand, there exists a closed hyperball $B(V, r)$ of radius $r>0$ centred at $V$ such that $B(V, r) \cap$ $G \widehat{B}(S)=\emptyset$, which follows that $\operatorname{vol}[B(V, r) \cap G \widehat{B}(S)]=0$. On the other hand, $V$ is a vertex of the hyper-rectangle $\widehat{B}(G S)$, so $\operatorname{vol}[B(V, r) \cap \widehat{B}(G S)]>$ 0 . This leads to $\operatorname{vol}[G \widehat{B}(S)]<\operatorname{vol}[\widehat{B}(G S)]$, which is a contradiction of the above assumption that $\operatorname{vol}[G \widehat{B}(S)]=\operatorname{vol}[\widehat{B}(G S)]$. Thus, we can prove if $\operatorname{vol}[G \widehat{B}(S)]=\operatorname{vol}[\widehat{B}(G S)]$, then $G \widehat{B}(S)=\widehat{B}(G S)$.

Furthermore, if $G \widehat{B}(S)=\widehat{B}(G S)$, then $\operatorname{vol}[G \widehat{B}(S)]=\operatorname{vol}[\widehat{B}(G S)]$. Hence, we can conclude $\operatorname{vol}[G \widehat{B}(S)]=\operatorname{vol}[\widehat{B}(G S)]$, if and only if $G \widehat{B}(S)=\widehat{B}(G S)$. 
In addition, since $G$ is an invertible linear mapping and $\widehat{B}(S)$ is a convex set, we have $\operatorname{vol}[G \widehat{B}(S)]=\operatorname{abs}(\operatorname{det}(G)) \operatorname{vol}[\widehat{B}(S)]$. Hence, we have

$$
\operatorname{abs}(\operatorname{det}(G)) \operatorname{vol}[\widehat{B}(S)]=\operatorname{vol}[G \widehat{B}(S)] \leqslant \operatorname{vol}[\widehat{B}(G S)]
$$

where the second " = "holds if and only if $G \widehat{B}(S)=\widehat{B}(G S)$. It follows that $\operatorname{abs}(\operatorname{det}(G)) \operatorname{vol}[\widehat{B}(S)]=\operatorname{vol}[\widehat{B}(G S)]$, if and only if $G(\widehat{B}(S))=\widehat{B}(G S)$. Moreover, $G \widehat{B}(S)=\widehat{B}(G S)$ means $G \widehat{B}(S)$ is a bounding hyper-rectangle. By Lemma 1, for $G$ such that $\operatorname{abs}(\operatorname{det}(G)) \operatorname{vol}[\widehat{B}(S)]=\operatorname{vol}[\widehat{B}(G S)]$, there exists $G=D P$, which is a perfect solution. Therefore, we can conclude that $G$ such that $\operatorname{vol}[\widehat{B}(G S)]=\operatorname{abs}(\operatorname{det}(G)) \operatorname{vol}[\widehat{B}(S)]$ is a perfect solution.

\section{Step3}

In Step1, (16) indicates a perfect solution $\bar{G}$ is such that $\operatorname{vol}[\widehat{B}(\bar{G} S)]=$ $\operatorname{abs}(\operatorname{det}(\bar{G})) \operatorname{vol}[\widehat{B}(S)]$. Conversely, Step2 proves that when $\operatorname{vol}[\widehat{B}(G S)]=$ $\operatorname{abs}(\operatorname{det}(G)) \operatorname{vol}[\widehat{B}(S)], G$ is a perfect solution. Thus, we can conclude that if and only if $G$ is a perfect solution that the second " $=$ " of (21) will hold. Combining (21) and (5) into (4), we have for any $G$

$$
\frac{\operatorname{vol}[\widehat{\varepsilon}(G S)]}{\operatorname{vol}[\widehat{B}(G S)]} \leqslant \frac{\operatorname{abs}(\operatorname{det}(G)) \operatorname{vol}[\widehat{\varepsilon}(S)]}{\operatorname{abs}(\operatorname{det}(G)) \operatorname{vol}[\widehat{B}(S)]}=\frac{\operatorname{vol}[\widehat{\varepsilon}(S)]}{\operatorname{vol}[\widehat{B}(S)]},
$$

where if and only if $G$ is a perfect solution that the first " = "will hold. The right side of (22) is independent of $G$, and only dependent on the sources. In BSS, the sources have already existed and fixed when they are observed, so $(22)$ is a constant for any perfect solution $\bar{G}$. Finally, one can conclude that the contrast function (4) is maximized if and only if $G$ is a perfect solution.

\subsection{Remarks for the New Proof}

The above subsection presents an alternative proof of the contrast function (4) under P1), P3) and A1). Assumption P2) is not directly required although it was stated by Erdogan [19]. We further analyse A1) and P2). Let $\mathbb{V}_{\widehat{B}(S)}, \mathbb{V}_{S}$ and $\mathbb{V}_{S_{i,:}}$ denote the set of vertices of $\widehat{B}(S), \operatorname{Conv}(S)$ and $\operatorname{Conv}\left(S_{i,:}\right)$, respectively. For any real-valued vector $S_{i,:}, \mathbb{V}_{S_{i,:}}=\left[\min \left[S_{i,:}\right], \max \left[S_{i,:}\right]\right]^{T}$, where $i \in\{1, \cdots, p\}$. By definition, the set of vertices of $\widehat{B}(S)$ is the Cartesian product of the set of vertices of each source $S_{i,:}$, i.e., $\mathbb{V}_{\widehat{B}(S)}=$ $\mathbb{V}_{S_{1,:}} \otimes \cdots \otimes \mathbb{V}_{S_{p,:}}$. Additionally, A1) means that $\mathbb{V}_{\widehat{B}(S)}=\mathbb{V}_{S}$, so $\mathbb{V}_{S}=$ 
$\mathbb{V}_{S_{1,:}} \otimes \cdots \otimes \mathbb{V}_{S_{p,:}}$. On the other hand, it has been shown that P2) is equivalent to ext $\mathbb{S}_{\check{S}}=\operatorname{ext} \mathbb{S}_{\check{S}_{1,:}} \otimes \cdots \otimes \operatorname{ext} \mathbb{S}_{\check{S}_{p,:}}$, where ext $\mathbb{S}_{\check{S}_{i,:}}$ denotes the extreme points of the $i$ th source support set, and ext $\mathbb{S}_{\check{S}}$ comprises all vertices of the convex hull [17]. The support set is the set of points, for which the probability density function is non-zero. For real-valued signals, the extreme point is equivalent to the vertex. Therefore, A1) assumes that in the sample space, the vertices of the bounding hyper-rectangle of $S$ should be observed. In contrast, P2) is a theoretical probabilistic assumption, and assumes that the vertices have positive probability. Thus, A1) is actually only possible if P2) is satisfied. Hence, the three assumptions P1), P3) and A1) imply the four assumptions P1)-P3) and A1).

Our proof has shown that elementary matrices and simple factorization of $G$ contribute to prove the contrast function (4). In this subsection, we analyse the imperfect solution by these techniques. In contrast to (15), for any imperfect solution $\underline{\mathrm{G}}$, the simply factorized product must contain at least one $E_{3}$ factor. Let $F_{3}$ denote the number of $E_{3}$ factors, and then we have

$$
\underline{G} \triangleq \prod_{f=1}^{F} E_{i}^{f}, i \in\{1,2,3\}, F_{3} \geqslant 1,
$$

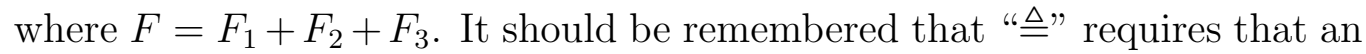
identity matrix and an elementary matrix should not be further factorized to two or more elementary matrices. The simply factorized product of $G$ is not unique, and a typical way to simply factorize is factorizing as the steps of Gauss-Jordan elimination.

Remark 1. Under assumptions $\boldsymbol{P 1}), \boldsymbol{P} 3)$ and $\boldsymbol{A 1})$, we can state that $\operatorname{vol}\left[\widehat{B}\left(E_{3} S\right)\right]>\operatorname{abs}\left(\operatorname{det}\left(E_{3}\right)\right) \operatorname{vol}[\widehat{B}(S)], \forall E_{3}$.

Proof. Recalling the definition of $E_{3}$ in (9), we get

$$
E_{3} S=E_{3}\left[\begin{array}{c}
\cdot \\
S_{u,:} \\
\cdot \\
S_{v,:} \\
\cdot
\end{array}\right]=\left[\begin{array}{c}
\cdot \\
S_{u,:}+k S_{v,:} \\
\cdot \\
S_{v,:} \\
\cdot
\end{array}\right] \text {. }
$$

From the above equation, $E_{3}$ only affects the $u$ th row of $S$, so we pay attention 
to the $u$ th row in the following. Substituting (9) into (6), we get

$$
\begin{aligned}
\operatorname{vol}\left[\widehat{B}\left(E_{3} S\right)\right] & =\prod_{i=1}^{p}\left\{\max \left[\left(E_{3} S\right)_{i,:}\right]-\min \left[\left(E_{3} S\right)_{i,:}\right]\right\} \\
& =\left\{\max \left[\left(E_{3} S\right)_{u,:}\right]-\min \left[\left(E_{3} S\right)_{u,:}\right]\right\} \prod_{i \neq u}\left\{\max \left[S_{i,:}\right]-\min \left[S_{i,:}\right]\right\}
\end{aligned}
$$

Under A1) that $S$ contains the vertices of its bounding hyper-rectangle, and as shown in Fig.1, we can be assured the following samples of $S$ (columns of $S$ ) exist:

$$
\begin{aligned}
& V_{m m}=\left[\cdots, \max \left[S_{u,:}\right], \cdots, \max \left[S_{v,:}\right], \cdots\right]^{T} \in S, \\
& V_{m n}=\left[\cdots, \max \left[S_{u,:}\right], \cdots, \min \left[S_{v,:}\right], \cdots\right]^{T} \in S, \\
& V_{n n}=\left[\cdots, \min \left[S_{u,:}\right], \cdots, \min \left[S_{v,:}\right], \cdots\right]^{T} \in S, \\
& V_{n m}=\left[\cdots, \min \left[S_{u,:}\right], \cdots, \max \left[S_{v,:}\right], \cdots\right]^{T} \in S . \\
\therefore \quad & \max \left[\left(E_{3} S\right)_{u,:}\right]-\min \left[\left(E_{3} S\right)_{u,:}\right] \\
= & \left\{\begin{array}{l}
\max \left[S_{u,:}\right]+k \max \left[S_{v,:}\right]-\min \left[S_{u,:}\right]-k \min \left[S_{v,:}\right], k>0 \\
\max \left[S_{u,:}\right]+k \min \left[S_{v,:}\right]-\min \left[S_{u,:}\right]-k \max \left[S_{v,:}\right], k<0
\end{array}\right. \\
= & \max \left[S_{u,:}\right]-\min \left[S_{u,:}\right]+\operatorname{abs}(k)\left\{\max \left[S_{v,:}\right]-\min \left[S_{v,:}\right]\right\} .
\end{aligned}
$$

Under P1), $\max \left[S_{i,:}\right]-\min \left[S_{i,:}\right]>0, \quad i \in\{1, \cdots, p\}$. Additionally, the definition of $E_{3}$ implies $k \neq 0$. Hence, (25) leads to

$$
\max \left[\left(E_{3} S\right)_{u,:}\right]-\min \left[\left(E_{3} S\right)_{u,:}\right]>\max \left[S_{u,:}\right]-\min \left[S_{u,:}\right]
$$

Substituting (26) into (24), and given $\operatorname{abs}\left(\operatorname{det}\left(E_{3}\right)\right)=1$, we can state more generally that $\operatorname{vol}\left[\widehat{B}\left(E_{3} S\right)\right]>\operatorname{abs}\left(\operatorname{det}\left(E_{3}\right)\right) \operatorname{vol}[\widehat{B}(S)]$.

Fig.1 illustrates Remark 1 as $p=2$, for visibility and without loss of generality. As shown on the left side of Fig.1, the region contoured by the bounding hyper-rectangle (the black rectangle) is the same as the convex hull (the green rectangle), which shows that under A1) $\widehat{B}(S)=\operatorname{Conv}(S)$ and $\operatorname{vol}[\widehat{B}(S)]=\operatorname{vol}[\operatorname{Conv}(S)]$. A shear mapping $E_{3}$ reshapes the convex hull (the green rectangle) on the left side to another convex hull (the green parallelogram) on the right side, and the volume of the two convex hulls are 


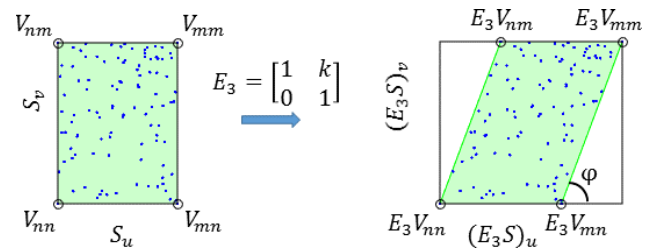

Figure 1: Illustration of Remark 1. The blue dots represent the samples, the regions enclosed by the black rectangles represent the bounding hyper-rectangles, and the green regions represent the convex hulls. On the left side, $S$ contains four vertices expressed by black circles. On the right side, the green parallelogram represents the reshaped bounding hyper-rectangle by $E_{3}$, and also is the convex hull of the mapped samples.

the same, i.e., $\operatorname{vol}[\operatorname{Conv}(S)]=\operatorname{vol}\left[\operatorname{Conv}\left(E_{3} S\right)\right]$. Note that on the right side, corresponding edges are tilted with a shear angle $\varphi$ by $E_{3}$, so these orthogonal edges become oblique edges. The shear factor $k$ is the cotangent of $\varphi$. Due to the shear angle $\varphi$, the maximum and minimum operations of the bounding hyper-rectangle (the black rectangle) expand the parallelogram $\operatorname{Conv}\left(E_{3} S\right)$ (the green parallelogram) to a new bounding hyper-rectangle $\widehat{B}\left(E_{3} S\right)$ (the black rectangle). Then, $\operatorname{vol}\left[\widehat{B}\left(E_{3} S\right)\right]>\operatorname{vol}\left[\operatorname{Conv}\left(E_{3} S\right)\right]=\operatorname{vol}[\widehat{B}(S)]$, as shown that the bounding hyper-rectangle (the black rectangle) on the right side is larger than that on the left side.

From a geometric point of view, since the edges of the bounding hyperrectangle are parallel to the coordinate axes, any edge of the bounding hyperrectangle is orthogonal to other edges that are connected at the same vertex. The shear mapping $E_{3}$ tilts the corresponding edges from orthogonal to oblique, while the reflection mapping $E_{1}$ and the scale mapping $E_{2}$ preserve the orthogonality of each pair of edges. These geometric features lead to the inequality of Remark 1 and the equalities of (10) and (11). Combining with the simple factorization of $G$ in (15) and (23), the inequality of (21) can be interpreted by the geometric features of elementary matrices.

\section{Further Analysis}

\subsection{Analysis of Assumptions}

In this subsection, we discuss the constraints in practical applications required by the four assumptions. P1) requires the amplitude of each source varying over a bounded and closed range. Realistic sources randomly vary in time or space over certain ranges, for example audio signals. Therefore, assumption P1) can be regarded as weak. In fact, many other BSS algorithms 
also require P1) implicitly. For instance, ICA algorithms use the higher order statistics $(\mathrm{HOS})^{3}$ of the sources, which implies the HOS of the sources is bounded. It follows that the sources are bounded, so ICA algorithms also assume P1) implicitly. Assumption P3) is a standard BSS assumption. Thus, we turn our attention to the assumptions A1) and P2).

In [19], the BCA algorithm was shown to have the potential advantage of performance improvement for short data records, and this advantage was supported by numerical experiments of artificial sources with Copula-t distributions of 10 sources and 5000 samples. The sources with Copula-t distributions are continuous variables, and this is a good example to evaluate the BCA algorithm.

However, we consider the other aspect of the $\mathrm{BCA}$ algorithm from the constraint of A1). For short records of continuous variables, A1) is a stringent assumption, in particular for tailed distributions ${ }^{4}$. Firstly, the vertex number of the bounding hyper-rectangle grows exponentially with the number of the sources $p$, that is $2^{p}$. Secondly, A1) implies that each edge of the convex hull is orthogonal to other edges connected with the same vertex, as the name "rectangle" suggests. For tailed distributions, the probability of each source attaining the extreme points is low, so the probability of more sources attaining their extreme points at the same sample are much lower.

Let us take audio signals as an example to analyse A1). As shown in Fig.2, for two pairs of audio signals $(p=2)$, the black rectangles represent the bounding hyper-rectangles which are external to the respective convex hulls, and neither the pair of speech signals ${ }^{5}$ nor the pair of music solos ${ }^{6}$ contains any vertex of their bounding hyper-rectangles. Even if the length of audio sources are increased, A1) is still stringent. To demonstrate this, we take one pair of 20-minute Obama talks ${ }^{7}$ which are shown on the left side of Fig.3, and one pair of 9-minute music solos ${ }^{8}$ which are illustrated on the right side. Similar to Fig.2, neither the pair of speech signals nor the pair

\footnotetext{
${ }^{3} \mathrm{HOS}$ refers to functions which use the higher power of a sample. The third and higher moments, as used in the skewness and kurtosis, are examples of HOS.

${ }^{4}$ Roughly speaking, a tailed distribution is a probability distribution with large skewness or kurtosis, compared to the normal distribution or the exponential distribution.

${ }^{5}$ TIMIT database

${ }^{6} \mathrm{https}$ ///archive.org/details/solo-piano-7

${ }^{7}$ http://www.obamadownloads.com/obama-mp3s.html

${ }^{8}$ http://www.mfiles.co.uk/classical-mp3.htm
} 

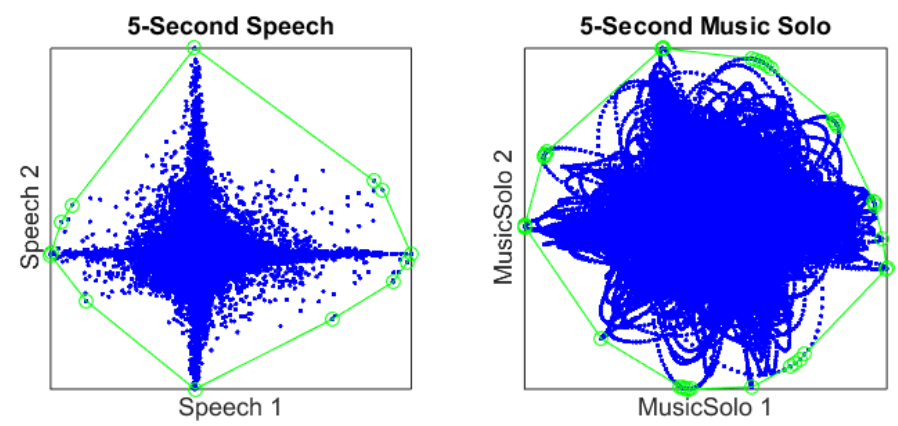

Figure 2: Scatter plot of two pairs of realistic audio signals. Two speech signals sampled at $16 \mathrm{KHz}$ for $5 \mathrm{~s}$ are on the left side, while two music solos sampled at $44.1 \mathrm{KHz}$ for $5 \mathrm{~s}$ are on the right side. The blue dots represent samples, the black rectangles represent the corresponding bounding hyper-rectangles, and the green lines with green circles represent the corresponding convex hulls.

of music solos contains any vertex of their bounding hyper-rectangles. Since A1) requires the sources to contain all of vertices, and the vertex number grows exponentially with the number of the sources, A1) can be considered stringent even for long duration of audio sources.
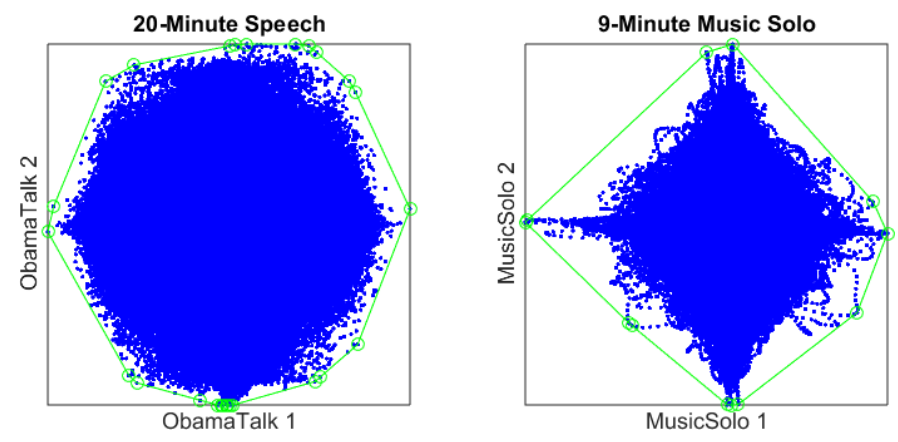

Figure 3: Scatter plot of long audio signals. Two speech signals sampled at $24 \mathrm{KHz}$ for $20 \mathrm{~m}$ are on the left side, while two music solos sampled at $44.1 \mathrm{KHz}$ for $9 \mathrm{~m}$ are on the right side. The blue dots represent samples, the black rectangles represent the corresponding bounding hyper-rectangles, and the green lines with green circles represent the corresponding convex hulls.

Although the pairs of audio signals are simplistic, these represent the fundamental signals which posed the BSS problem as the so-called "cocktail party problem", and audio signals have been one of the main applications of BSS over the past three decades. 


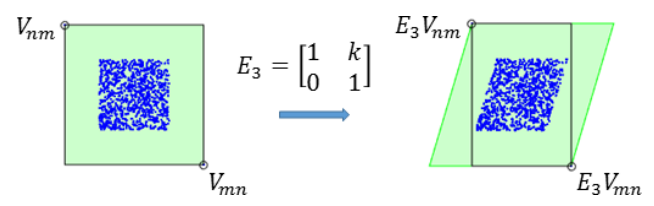

Figure 4: A simple example that $E_{3}$ (shear mapping) makes the bounding hyper-rectangle of tailed distributions shrink, given A1) is unsatisfied. The blue dots and the black rectangles represent the samples of the corresponding bounding hyper-rectangles, respectively. On the left side, $S$ only contains two vertices $V_{m n}$ and $V_{n m}$, expressed by black circles. On the right side, $E_{3} V_{m n}$ and $E_{3} V_{n m}$ are the respective vertices mapped by $E_{3}$ left multiplication, and the green parallelogram is the bounding hyper-rectangle reshaped by $E_{3}$.

On the basis of the above discussion, it is reasonable to assume that for BSS problems on tailed distributions, P1) and P3) are satisfied, but A1) is unsatisfied. In such conditions, (10) and (11) still hold, and it follows that (16) still holds. Therefore, even though A1) is unsatisfied, under P1) and P3) the contrast function (4) is preserved for the perfect solution $\bar{G}$. Comparing (15) and (23), we can conclude that the key difference between the perfect solution and the imperfect solution is the $E_{3}$ factor.

For $E_{3}$ given $\left.\mathbf{A} 1\right)$ is unsatisfied, Remark 1 does not hold. It follows that there likely exists an $E_{3}$ such that $\operatorname{vol}\left[\widehat{B}\left(E_{3} S\right)\right]<\operatorname{vol}[\widehat{B}(S)]$. For such a condition, since $\operatorname{abs}\left(\operatorname{det}\left(E_{3}\right)\right)=1$, we get

$$
\frac{\operatorname{vol}\left[\widehat{\varepsilon}\left(E_{3} S\right)\right]}{\operatorname{vol}\left[\widehat{B}\left(E_{3} S\right)\right]}=\frac{\operatorname{abs}\left(\operatorname{det}\left(E_{3}\right)\right) \operatorname{vol}[\widehat{\varepsilon}(S)]}{\operatorname{vol}\left[\widehat{B}\left(E_{3} S\right)\right]}>\frac{\operatorname{vol}[\widehat{\varepsilon}(S)]}{\operatorname{vol}[\widehat{B}(S)]}
$$

By comparing (27) and (22), the $G$ that is obtained by maximizing (4) will contain at least one $E_{3}$ factor, and thus that $G$ is an imperfect solution. As a result, the BCA algorithm based on (4) will fail, given such $E_{3}$ exists.

Given A1) is unsatisfied, from a geometric point of view, if there exists an $E_{3}$ such that it makes the bounding hyper-rectangle of $S$ shrink, the $G$ obtained by maximizing (4) will be an imperfect solution. For visibility and without loss of generality, we provide a simple two dimensional $(p=2)$ example to demonstrate that for tailed distributions there likely exists an $E_{3}$ that makes vol $[\widehat{B}(S)]$ shrink. Consider two outliers $V_{n m}=[-2 c, 2 c]^{T}, V_{m n}=$ $[2 c,-2 c]^{T}, c>0$, representing the tails of an otherwise uniform distribution in the range $[-c, c]$. It follows that $S$ only contains two out of the four vertices of its bounding hyper-rectangle. As Fig. 4 shows, the $E_{3}$ with $k=0.3$ makes the bounding hyper-rectangle shrink from $16 c^{2}$ to $11.2 c^{2}$. 
The existence of such an $E_{3}$ is determined by the distribution of sources. For tailed distributions, e.g., audio signals, the performance of BCA contrast function (4) is likely to suffer, since A1) is likely to be unsatisfied.

Based on our new proof, we further analyse that the instantaneous BCA algorithm is likely to suffer for tailed distributions, for instance audio signals, because A1) is a stringent assumption for continuous sources of tailed distributions. It was briefly stated by Erdogan in conclusion [21] that the performances of the convolutive BCA algorithm is likely to suffer for tailed distributions. Our analysis indicates this limitation is also for the originally instantaneous BCA and is likely to extend to other cases which rely on A1). Although the BCA algorithm is termed as "bounded", the key assumption of BCA in practical applications is A1) (or associated P2)), rather than the bounded assumption P1).

\begin{tabular}{||c|c||}
\hline BCA assumptions & ICA assumptions \\
\hline \hline Bounded P1)(explicitly) & Bounded P1)(implicitly) \\
\hline Determined P3) & Determined P3) \\
\hline Geometric A1) & Stochastic (Statistical Independence) \\
\hline
\end{tabular}

Table 1: Assumptions of BCA and ICA

Although ICA algorithms do not explicitly require that the sources are bounded, ICA algorithms use HOS and calculate HOS based on samples. Hence, ICA algorithms require that HOS of the sources are bounded. It follows that ICA algorithms implicitly assume the sources are bounded. In [19], BCA was considered as a more general approach, covering ICA as a special case for bounded sources. However, we consider that BCA is established based on the geometric assumption A1), while ICA is established based on the stochastic assumption (Statistical Independence), in addition to the bounded assumption P1) and the determined assumption P3).

Fundamentally, BCA and ICA are different from the geometric assumption A1) and the stochastic assumption Statistical Independence, as shown in Table 1. As Fig.5 illustrates, there is an intersection where A1) is unsatisfied and independent. Some audio sources can be considered independent but not satisfying $\mathbf{A 1}$ ), which will be shown in the next subsection. Therefore for bounded sources, BCA can be considered as a complementary approach of ICA, rather than a more general approach than ICA. 


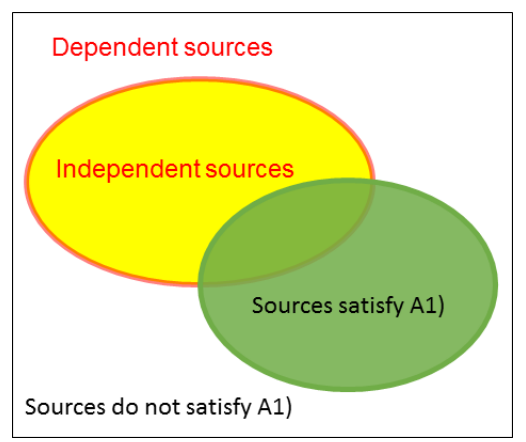

Figure 5: Illustration of the sources divided by the geometric assumption A1) and the stochastic assumption Statistical Independence. The sources within the green oval satisfy A1), while the sources within the red oval satisfy Statistical Independence.

\subsection{Numerical Experiments}

In order to investigate our theoretical analysis, we performed simulations on audio sources. We evaluated the BCA algorithm [19] using the MATLAB code $^{9}$ provided by Erdogan [19]. Benchmarks were ICA algorithms: FastICA [11], JADE [9] and SOBI [7]. One evaluation criterion is a classical BSS performance index (PI) for the overall mapping $G=W H[26]$ as

$$
\begin{aligned}
P I(G)= & \frac{1}{2 p(p-1)}\left\{\sum_{i=1}^{p}\left(\sum_{j=1}^{p} \frac{|G(i, j)|^{2}}{\max _{k}|G(i, k)|^{2}}-1\right)\right. \\
& \left.+\sum_{j=1}^{p}\left(\sum_{i=1}^{p} \frac{|G(i, j)|^{2}}{\max _{k}|G(k, j)|^{2}}-1\right)\right\} .
\end{aligned}
$$

The other criterion is the sum square error (SSE) for the separated sources $[27]$ as

$$
S S E(S, W Y)=\frac{1}{p} \min _{\pi \in \Pi_{p}} \sum_{i=1}^{p}\left\|\frac{S_{i,:}}{\left\|S_{i,:}\right\|}-\frac{(W Y)_{\pi_{i}}}{\left\|(W Y)_{\pi_{i}}\right\|}\right\|^{2},
$$

where $\pi=\left(\pi_{1}, \ldots, \pi_{p}\right)^{T}$, and $\prod_{p}=\left\{\pi \in \mathbb{R}^{p \times 1} \mid \pi_{i} \neq \pi_{j}, \forall i \neq j\right\}$ is the set of all permutations of $\{1,2, \ldots, p\}$. The permutation ambiguity is resolved by the Hungarian algorithm [28] and the authors offer MATLAB

\footnotetext{
${ }^{9}$ http://aspc.ku.edu.tr/bcasoftware.html
} 
source code ${ }^{10}$ online. The scaling ambiguity is normalized by Euclidean norm like $S_{i,:} /\left\|S_{i,:}\right\|$. Directly, for the PI and SSE indices the smaller they are, the better the separation quality.

Four experiments were conducted on realistic speech sources and music sources separately. In each experiment, 500 independent runs were performed and averaged. For each independent run, the observed signals $Y$ are artificially generated by randomly selected sources and the mixing matrix $H$, which was generated randomly from a zero-mean unit-variance normal distribution.
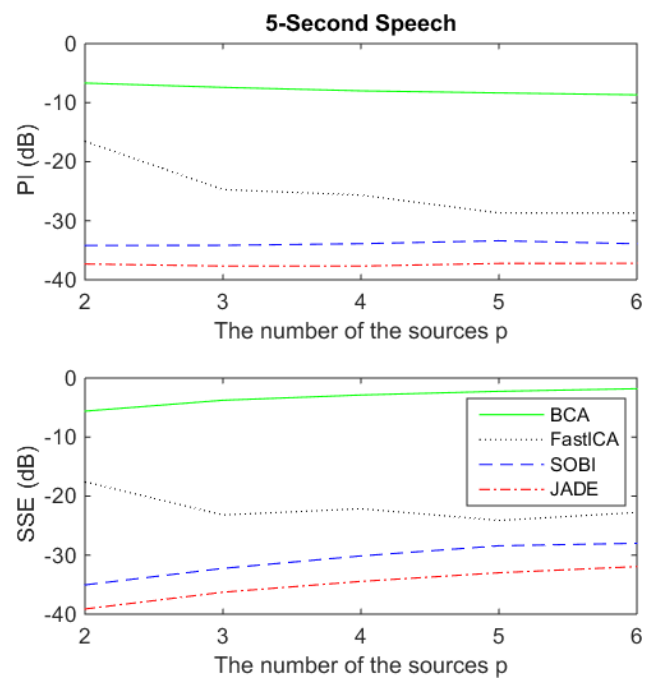

Figure 6: Mean SSE and PI for 5-second speech sources by the number of sources $\mathrm{p}$

The first experiment was conducted on 50 speech segments from the TIMIT dataset, which were sampled at $16 \mathrm{KHz}$ and truncated for 5 seconds. Fig.6 compares, the performance of the three ICA algorithms and BCA algorithm, by the PI and SSE for different number of sources $p=2, \ldots, 6$. Although the performance of FastICA was worse than the other two ICA algorithms, the three ICA algorithms all achieved satisfactory separation performance. In fact, the determined instantaneous BSS problems on speech sources have been well-developed [2]. However, the separation performance of the BCA algorithm suffered. This result supports our theoretical analysis in the Section 3.1.

\footnotetext{
${ }^{10} \mathrm{http}: / /$ itakura.ite.tul.cz/zbynek/tddeconv.htm
} 

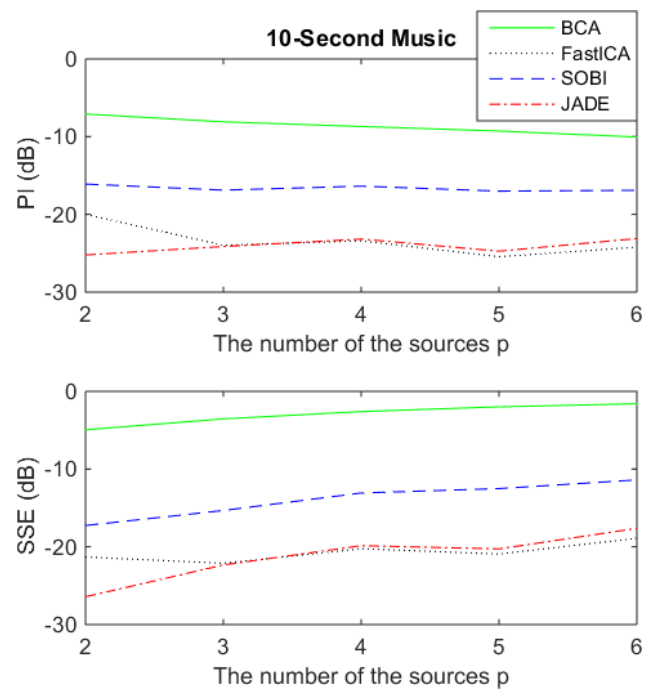

Figure 7: Mean SSE and PI for 10-second music solos by the number of sources $\mathrm{p}$

The second experiment evaluated the PI and SSE on 50 piano $^{11}$ and violin $^{12}$ segments, which were sampled at $44.1 \mathrm{KHz}$ and truncated for 10 seconds. Fig.7 illustrates the PI and SSE of 10-second music solos separated by the BCA algorithm against the ICA benchmarks for different number of the sources $p=2, \ldots, 6$. We can also see that the three ICA algorithms outperformed the BCA algorithm on the two criteria, although there were performance gaps between the ICA algorithms. The performance gaps between the BCA algorithm and the worst benchmark are all approximate 10 $\mathrm{dB}$. This result also underpins our theoretical analysis in the above subsection.

Furthermore, to study the separation performance for longer music sources, we conducted the third experiment on the 50 piano and violin segments truncated for 100 seconds. Fig.8 depicts the PI and SSE of 100-second music solos separated by the BCA algorithm against benchmarks for different number of sources $p=2, \ldots, 6$. We can also see that the three ICA algorithms also outperformed the BCA algorithm on the two criteria. Compared with the separation performance of BCA for 10-second music solos as shown in Fig.7, there was not any substantial improvement for the separation performance

\footnotetext{
${ }^{11}$ https://archive.org/details/solo-piano-7

${ }^{12}$ http://www.tasminlittle.org.uk
} 

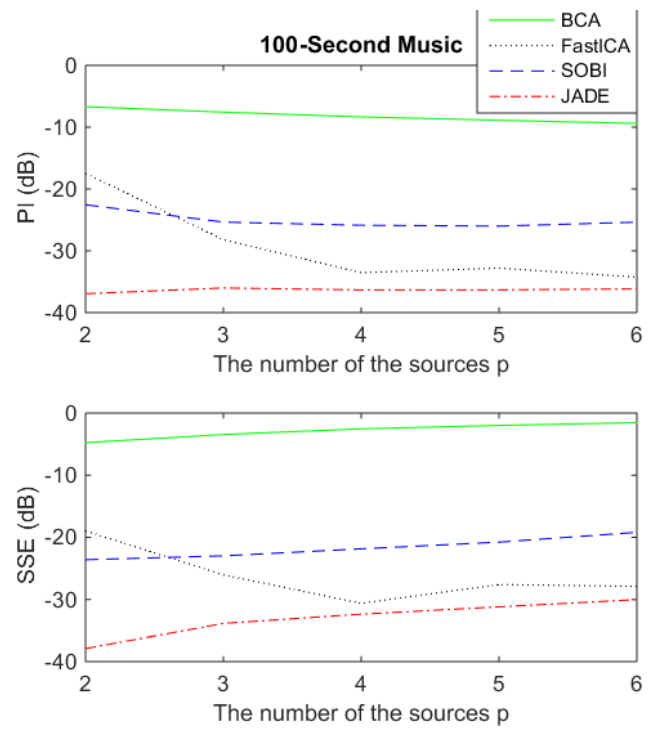

Figure 8: Mean SSE and PI for 100-second music solos by the number of sources $\mathrm{p}$ on tenfold length music solos.
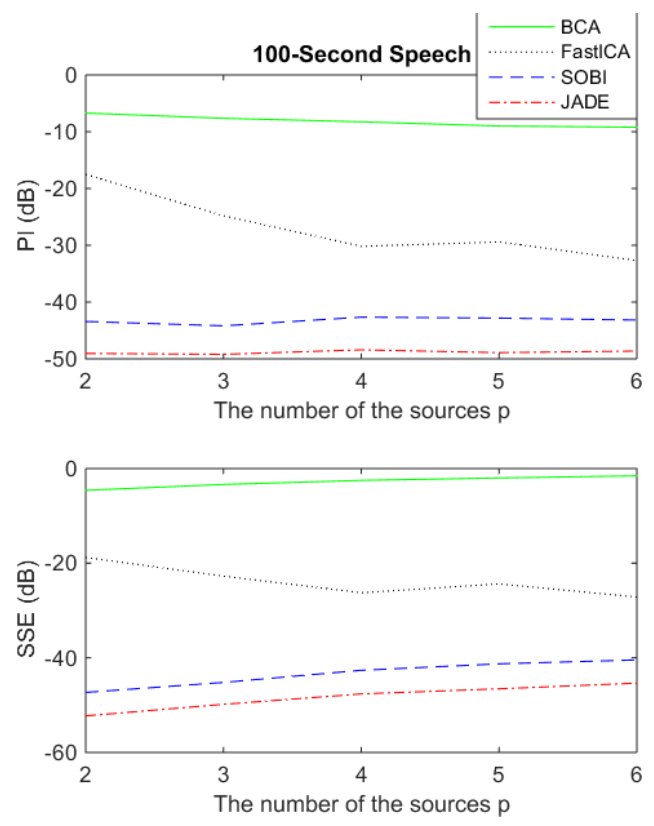

Figure 9: Mean SSE and PI for 100-second speech sources by the number of sources $\mathrm{p}$ 
Similarly, we conducted the fourth experiment on longer speech sources. 65 speech segments ${ }^{13}$ which were sampled at $24 \mathrm{KHz}$ and truncated for 100 seconds. Fig.9 depicts the PI and SSE of 100-second speech sources separated by the BCA algorithm against benchmarks for different number of sources $p=2, \ldots, 6$. The performance gaps between the BCA algorithm and the worst benchmark are also significant. Compared with the separation performance of BCA for 5-second speech sources as shown in Fig.6, there was not any substantial improvement for the separation performance on twenty-fold length speech sources.

To conclude, the four numerical experiments show that the ICA algorithms outperformed the $\mathrm{BCA}$ algorithm. These results support our view that the BCA algorithms based on A1) will suffer for continuous taileddistributed sources, and there are some bounded sources that can be considered independent but not satisfying A1), e.g. audio sources. We have taken audio signals as examples, but the BCA algorithms are also likely to suffer on other tailed distribution signals, such as electroencephalography (EEG) signals, and magnetoencephalography (MEG) signals.

\section{Conclusion}

This paper presents a new proof of Erdogan's BCA contrast function (4), based on the basic principles of linear algebra and convex geometry. The analysis framework derived from elementary matrices and Gauss-Jordan elimination can be potentially useful in other settings of BSS. Moreover, the link between the key assumptions A1) and P2) is provided. This paper analyses that A1) can be considered as a stringent assumption for continuous sources with tailed distributions, and that $\mathbf{A 1}$ ) is stringent in some practical applications, where the performances of the BCA contrast function are likely to suffer. For example, audio sources can be considered are bounded and independent but not satisfying A1). Numerical experiments on audio sources support our analysis. Hence for bounded sources, BCA can be considered as a complementary approach to ICA, not a more general approach than ICA.

\footnotetext{
${ }^{13}$ http://www.obamadownloads.com/obama-mp3s.html
} 


\section{References}

[1] A. Hyvarinen, J. Karhunen, E. Oja, Independent component analysis, J. Wiley, New York, 2001.

[2] P. Comon, C. Jutten, Handbook of Blind Source Separation: Independent Component Analysis and Applications, Elsevier Science, Burlington, MA, USA, 2010.

[3] J. F. Cardoso, Blind signal separation: Statistical principles, IEEE Proc. 86 (10) (1998) 2009-2025.

[4] B. Ans, J. Herault, C. Jutten, Adaptive neural architectures: Detection of primitives, in Proc. COGNITIVA (1985) 593-597.

[5] J. Herault, C. Jutten, Space or time adaptive signal process. by neural network models, Intern. Conf. on Neural Networks for Computing (1986) 206-211.

[6] D. T. Pham, Blind separation of instantaneous mixture of sources via an independent component analysis, IEEE Trans. Signal Process. 44 (11) (1996) 2768-2779.

[7] A. Belouchrani, K. AbedMeraim, J. F. Cardoso, E. Moulines, A blind source separation technique using second-order statistics, IEEE Trans. Signal Process. 45 (2) (1997) 434-444.

[8] L. De Lathauwer, B. De Moor, J. Vandewalle, Independent component analysis and (simultaneous) third-order tensor diagonalization, IEEE Trans. Signal Process. 49 (10) (2001) 2262-2271.

[9] J. F. Cardoso, A. Souloumiac, Blind beamforming for non-gaussian signals, IEE Proc. F (Radar and Signal Process.) 140 (6) (1993) 362-370.

[10] J.-F. Cardoso, On the performance of orthogonal source separation algorithms, Proc. EUSIPCO (1994) 776-779.

[11] A. Hyvarinen, Fast and robust fixed-point algorithms for independent component analysis, IEEE Trans. Neural Netw. 10 (3) (1999) 626-634. 
[12] D. T. Pham, J. F. Cardoso, Blind separation of instantaneous mixtures of nonstationary sources, IEEE Trans. Signal Process. 49 (9) (2001) $1837-1848$.

[13] P. W. Chen, H. Hung, O. Komori, S. Y. Huang, S. Eguchi, Robust independent component analysis via minimum gamma-divergence estimation, IEEE J. Sel. Topics Signal Process. 7 (4) (2013) 614-624.

[14] F. Vrins, J. A. Lee, M. Verleysen, A minimum-range approach to blind extraction of bounded sources, IEEE Trans. Neural Netw. 18 (3) (2007) 809-822.

[15] A. T. Erdogan, A simple geometric blind source separation method for bounded magnitude sources, IEEE Trans. Signal Process. 54 (2) (2006) 438-449.

[16] W. Gao, R. Togneri, V. Sreeram, A contrast function and algorithm for blind separation of audio signals, Proc. Interspeech (2017) 1889-1893.

[17] S. Cruces, Bounded component analysis of linear mixtures: A criterion of minimum convex perimeter, IEEE Trans. Signal Process. 58 (4) (2010) 2141-2154.

[18] S. Cruces, Bounded component analysis of noisy underdetermined and overdetermined mixtures, IEEE Trans. Signal Process. 63 (9) (2015) 2279-2294.

[19] A. T. Erdogan, A class of bounded component analysis algorithms for the separation of both independent and dependent sources, IEEE Trans. Signal Process. 61 (22) (2013) 5730-5743.

[20] H. A. Inan, A. T. Erdogan, A convolutive bounded component analysis framework for potentially nonstationary independent and/or dependent sources, IEEE Trans. Signal Process. 63 (1) (2015) 18-30.

[21] H. A. Inan, A. T. Erdogan, Convolutive bounded component analysis algorithms for independent and dependent source separation, IEEE Trans. Neural Netw. Learn. Syst. 26 (4) (2015) 697-708.

[22] E. Babatas, A. T. Erdogan, Sparse bounded component analysis, Machine Learning for Signal Processing (MLSP), 2016 IEEE 26th International Workshop (2016) 1-6. 
[23] H. A. Inan, A. T. Erdogan, S. Cruces, Stationary point characterization for a class of bca algorithms, IEEE Trans. Signal Process. 65 (20) (2017) $5437-5452$.

[24] B. Grünbaum, Convex Polytopes, Springer-Verlag, New York, USA, 2003.

[25] P. Gruber, J. Wills, Handbook of Convex Geometry, Elsevier Science, Amsterdam, The Netherlands, 1993.

[26] A. Boudjellal, A. Mesloub, K. Abed-Meraim, A. Belouchrani, Separation of dependent autoregressive sources using joint matrix diagonalization, IEEE Signal Proc. Lett. 22 (8) (2015) 1180-1183.

[27] Z. Y. Yang, Y. Xiang, Y. Rong, K. Xie, A convex geometry-based blind source separation method for separating nonnegative sources, IEEE Trans. Neural Netw. Learn. Syst. 26 (8) (2015) 1635-1644.

[28] P. Tichavsky, Z. Koldovsky, Optimal pairing of signal components separated by blind techniques, IEEE Signal Proc. Lett. 11 (2) (2004) 119122 . 